\title{
Adjuvant traditional chinese medicine qingyi decoction on severe acute pancreatitis
}

\author{
Hong-yang Deng*, Jian Dü, Zi-wei Shao, Wen-qiang Xie, Wei-jia Cao, Feng-xian Wei* and Xiao-dong Xu* \\ Lanzhou University Second Hospital, Lanzhou 730030, China \\ \#Contributed equally
}

\begin{abstract}
Acute pancreatitis (AP) is a self-digestive disease and one of the clinical acute abdominal diseases. Severe AP is associated with severe morbidity and mortality, and its comprehensive treatment involves medication, surgery and complementary therapies. In China, Traditional Chinese medicine (TCM) is one of the most popular complementary and alternative choices for AP Current both basic and clinical researches show that Qingyi decoction has effective effect on AP. This article reviewed the possible mechanisms of widely used Chinese Qingyi decoction and its main activity ingredients (rhubarb and emodin) in the adjuvant treatment of AP. As it is rich, low cost and safe as well as other TCM, Qingyi decoction would be used to deal with various pathogenesis, which can achieve potentially better clinical benefits and more choices for AP treatment in the future.
\end{abstract}

\section{Short communication}

Severe acute pancreatitis (SAP) is one of the most common acute abdomens of alimentary system. AP refers to the activation of trypsin caused by multiple causes, local inflammation of pancreas as the main feature, and it may affect other organ functions. Mostly, SAP can induce Systemic inflammatory response syndrome (SIRS) and Multiple organ dysfunction syndrome (MODS) in a short period of time. Thus, its clinical process is extremely dangerous. Currently, clinical diagnosis of AP in guidelines of both the revised Atlanta classification (RACAP) and classification of acute pancreatitis severity (DBCAPS) is mainly based on its radiology. Its treatment is focused to inhibit pancreatic and gastrointestinal function and secretion, and to down-regulate the level of antibacterial and anti-inflammatory in body, and also relieve abdominal pain abdominal distension [1].

In China, Traditional Chinese medicine (TCM) is also important adjuvant therapy in the clinical practice for SAP. The combined treatment of TCM can improve the cure rate, yet the death rate is still as high as $20 \%$ [2]. In recent years, Qingyi decoction and its monarch medicine Rhubarb have been proved to be effective in antiinflammatory, bacteriostatic, abdominal distension and abdominal pain relief in pharmacological experiments and clinical studies [3]. It has been reported that Qingyi decoction can be used as an effective drug after administration through gastric tube or retention enema, it can alleviate the patient's symptoms and improve the development of the condition. Pre-clinical and clinical studies have proved its efficacy and more and more researches reveal the underlying mechanisms. Firstly, by targeting the inflammatory response of SAP, Qingyi decoction decreased the levels of serum endotoxin, $\alpha$-amylase and tumor necrosis factor- $\alpha$, and decreased the expression of inflammatory factors. It can also decrease the severeity of the intestinal barrier injury and lung injury caused by SAP through decreasing the level of expression of phospholipase A2 in intestine and lung [4,5]. Qingyi decoction can also increase the intestinal mucosal density, height and area, so this would be helpful to upregulate the level of intestinal histone, to inhibite intestinal permeability, and therefore to inhibit intestinal bacterial translocation. After that, Qingyi decoction combined with enteral nutrition would improve clinical efficacy through improving intestinal function and inflammatory cytokines [6].

Rhubarb is the main agents in Qingyi decoction, and it also has a plenty of other agents such as Radix Paeoniae Alba, Scutellaria baicalensis, and Coptis chinensis. In animal studies, Rhubarb was reported to play important roles in triggering intestinal peristalsis, preventing intestinal bacterial translocation, regulating intestinal flora, and repairing intestinal mucosal barrier in SAP. Emodin is the main activity compound in Rhubarb with a molecular formula of $\mathrm{C} 15 \mathrm{H} 10 \mathrm{O} 5$ and molecular weight of 270.23, and its signal pathway was found to be through Toll like receptors (TLR) 2 and 4 down-regulation [7]. Wan et al reported the effective effects of early enteral nutrition combined with rhubarb in a randomized controlled trial, and the results showed that combined use of nutrition therapy and rhubarb significantly reduced the white blood cell count, plasma C-reactive protein and IL-6 levels and increased the plasma IL-11 levels [8]. what's more, recovery time of abnormal defecation, high fever, intensive care unit and hospitalization were all shortened after adjuvant rhubarb administration. As the levels of plasma alanine aminotransferase, aspartate aminotransferase and creatinine $(\mathrm{Cr})$ in combination group was also decreased, and the authors proposed that rhubarb may also alleviate the liver and kidney dysfunction commonly associated with SAP. Zhou et al studied the

${ }^{*}$ Correspondence to: Xiao-dong $\mathrm{Xu}$, Cuiyingmen no.82, Lanzhou University Second Hospital, Chengguan District, Lanzhou 730030, China, E-mail: 13893273850@163.com

Feng-xian Wei, Lanzhou University Second Hospital, Lanzhou 730030, China, E-mail: weifx08@126.com

Key words: acute pancreatitis, qingyi decoction, rheum, emodin

Received: October 24, 2018; Accepted: October 31, 2018; Published: November 05,2018 
combined effects of rhubarb and somatostatin in AP patients, the results showed that rhubarb significantly reduced the total complications and APACHE II score of SAP patients, which supported that rhubarb can be used as an effective adjuvant ingredient for SAP.

\section{Conclusion}

Qingyi decoction was reported to be effective in AP adjuvant treatment by both basic research and clinical studies. Sufficient evidence mainly reveled the underlying mechanism of its main activity ingredient of Rhubarb and Emodin. However, as a TCM decoction there was so many kinds of Chinese medicine and unclear ingredient and compound existed, it is hard to separate and study them as single activity ingredient. More studies on its safety is warranted to ensure its proper clinical application.

\section{References}

1. Zhou Y, Wang L, Huang X, Li H, Xiong Y (2016) Add-on effect of crude rhubarb to somatostatin for acute pancreatitis: A meta-analy-sis of randomized controlled trials. $J$ Ethnopharmacol 194: 495-505. [Crossref]
2. Xu XD, Wang ZY, Zhang LY, Ni R, Wei FX, et al. (2015) Acute pancreatitis classifications: Basis and key goals. Medicine 94: e2182. [Crossref]

3. Wang X, Li Z, Yuan Y (2013) Diagnosis and treatment of acute pancreatic gland inflammation in China. Chinese Journal of practical Internal Medicine 7: 530-535.

4. Zhang JW, Zhang GX, Chen HL, Liu GL, Owusu L, et al. (2015) Therapeutic effect of Qingyi decoction in severe acute pancreatitis-induced intestinal barrier injury. World $J$ Gastroenterol 21: 3537-3546. [Crossref]

5. Weijia C, Fengxian W, Guohong S (2018) A meta-analysis: Qingyi decoction combined with somatostatin on acute pancreatitis. JETCM 27: 1542-1551.

6. Zhang B, Huang HT, Wang ZG, Rong YP, Yu B, et al. (2010) The effects of Qing Yi decoction in combination with enteral nutrition on inflammatory cytokine balance of patients with severe acute pancreatitis. Fubu Waike Zazhi 2: 108-110.

7. Yao P, Cui M, Li Y, Deng Y, Wu H (2015) Effects of rhubarb on intestinal flora and toll-like receptors of intestinal mucosa in rats with severe acute pancreatitis. Pancreas 44: 799-804. [Crossref]

8. Wan B, Fu H, Yin J, Xu F (2014) Efficacy of rhubarb combined with early enteral nutrition for the treatment of severe acute pancreatitis: A randomized controlled trial. Scand J Gastroenterol 49: 1375-1384. [Crossref]

Copyright: C2018 Deng H. This is an open-access article distributed under the terms of the Creative Commons Attribution License, which permits unrestricted use, distribution, and reproduction in any medium, provided the original author and source are credited. 